\title{
Supervivencia global del cáncer colorrectal metastásico en Lima Metropolitana: relación con el estado mutacional del gen KRAS
} Overall survival of metastatic colorectal cancer in Lima:
Relationship with the KRAS gene mutational status

Correspondencia

Franklin Aldecoa

franklin.aldecoa@yahoo.com

Recibido: 04/01/2020

Arbitrado por pares

Aprobado: 27/05/2020

Citar como: Aldecoa F.

Supervivencia global del cáncer colorrectal metastásico en Lima Metropolitana: relación con el estado mutacional del gen KRAS. Acta Med Peru. 2020;37(2):122-9. doi: https://doi.org/10.35663/ amp.2020.372.868

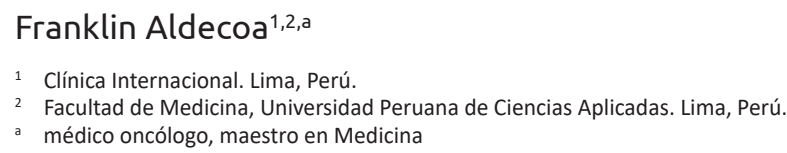

Este estudio fue elaborado con parte de la información proveniente de la tesis para optar el grado de maestro en Medicina: Adenocarcinoma colorrectal metastásico con estudio mutacional del gen K-ras (mutado versus no mutado) en el exón 2 (codones 12 y 13), en el Perú: análisis de los resultados con diferentes tipos de tratamiento.

\section{RESUMEN}

Objetivos: el objetivo principal del estudio fue evaluar la supervivencia global en pacientes con carcinoma colorrectal metastásico (CCRm) cuyos tumores tuvieran el gen KRAS mutado frente al no mutado. Materiales y métodos: se analizaron los datos de las historias clínicas de pacientes con CCRm (enero 2010 - diciembre 2013) de diferentes hospitales de Lima Metropolitana, cuyos tumores tuvieron evaluación del estado mutacional del exón 2, gen KRAS. Se usaron la curva de supervivencia de Kaplan-Meier y la prueba de long rank o Breslow para las comparaciones de las curvas de supervivencia. Resultados: de los 320 casos analizados, hubo 227 pacientes (70,93\%) con KRAS no mutado y $93(29,07 \%)$ con KRAS mutado. La supervivencia global de pacientes con CCRm y KRAS mutado fue mayor que los pacientes con KRAS no mutado (hazart ratio: 0,73 ; IC $95 \%$ 0,55-0,98; $p=0,037)$. Conclusión: la población con CCRm y KRAS mutado estudiada en centros médicos de Lima Metropolitana tuvo una supervivencia mayor, comparada con la no mutada, comportamiento diferente a lo encontrado en la literatura mundial.

Palabras clave: Cáncer colorrectal; /genética; Mutación; Exones (fuente: DeCS-BIREME).

\section{ABSTRACT}

Objectives: The main goal for this study was to evaluate overall survival in mCRC patients with mutated vs. wild type KRAS gene (exon 2) status. Materials and Methods: Between January 2010 and December 2013, data from clinical records of mCRC patients from different hospitals in Lima, stating an assessment of the KRAS gene mutation status (exon 2), were analyzed. Kaplan-Meier survival estimates and Log-Rank or Breslow Test were used to compare the survival curves. Results: Three-hundred and twenty cases were analyzed. There were 227 patients $(70.93 \%)$ with wild type KRAS and 93 (29.07\%) with mutated KRAS. The overall survival of mCRC patients and mutated KRAS was higher than that of patients with wild type KRAS (HR: $0.73 ; 95 \% \mathrm{Cl}: 0.55-0.98 ; \mathrm{P}=0.037$ ). Conclusion: Patients with $\mathrm{mCRC}$ and mutated KRAS who were studied in Lima have higher survival rates compared to wild type patients, being this different from what is found in the world literature.

Keywords: Cancer, colorectal; /genetics; Mutation; Exons (source: MeSH NLM). 


\section{INTRODUCCIÓN}

De acuerdo con el reporte de Registro de cáncer de Lima Metropolitana 2010-2012, la incidencia de cáncer colorrectal (CCR) fue de 14,43 casos por cada 100000 habitantes y representó el $6,7 \%$ de todas las neoplasias malignas, con una ligera predominancia del sexo femenino $(53,5 \%)^{[1]}$. Alrededor de $75 \%$ de los casos nuevos se presentaron esporádicamente; $y$ entre el 15 y el $20 \%$ de los casos tenía antecedente familiar de carcinoma colorrectal. Por otro lado, en general, la metástasis a distancia se diagnostica en aproximadamente el $20 \%$ de los pacientes en el momento del diagnóstico ${ }^{[2]} y$, aunque antes era vista como incurable, en entornos favorables la supervivencia media puede superar los cinco años en pacientes con metástasis hepáticas o pulmonares solitarias ${ }^{[3]}$.

La sobreexpresión del factor de crecimiento epitelial (EGFR) es característico en las células del CCR metastásico (CCRm) y está asociada a una pobre evolución clínica; además, se ha identificado que hasta $90 \%$ de los tumores metastásicos la presenta ${ }^{[4,5]}$.

En la actualidad, existen anticuerpos monoclonales que logran un efecto antitumoral inhibiendo la expresión del EGFR; este efecto se produciría mediante el bloqueo de múltiples vías de señalización (MAPK, fosfatidilinositol-3-cinasa, AKT, las cinasas JAK/STAT, proteína cinasa $C$, entre otras) que propician dicha expresión ${ }^{[6]}$. La proteína K-ras, responsable - entre otras- de la activación del EGFR, es producida por el gen KRAS que muta con más frecuencia dentro de estas vías de señalización, por lo que es considerada como un potencial biomarcador molecular en la terapia anti-EGFR ${ }^{[7]}$. Las mutaciones en el KRAS han sido encontradas entre el $35 \%$ y el $45 \%$ de casos con CCR y los codones 12 y 13 - dentro del exón 2- son los puntos más frecuentes de estas mutaciones (casi el $95 \%$ de todas las mutaciones) ${ }^{[8-11]}$.

En el Perú, la búsqueda de mutaciones del gen KRAS (exón 2) en casos con CCRm se realizó desde la segunda mitad del año 2008 hasta finales del año 2013, ya que, en setiembre de 2013, fue presentado un subanálisis del estudio FIRE-3, que comparaba los esquemas de primera línea FOLFIRI más cetuximab frente a FOLFIRI más bevacizumab en pacientes con tumores KRAS no mutado (German AIO study KRK-0306) ${ }^{[12]}$, y se demostró que extender la búsqueda a mutaciones en otros codones que no fueran el 2 en el gen KRAS y a mutaciones en los otros genes RAS, como NRAS, excluía a más pacientes que no se beneficiaban de una terapia anti-EGFR ${ }^{[13]}$. A pesar de que han pasado más de 30 años del descubrimiento del papel de KRAS en la transformación de células, aún no hay drogas dirigidas a atacar a sus mutaciones activadoras presentes en los pacientes con CCRm ${ }^{[14]}$.

La evaluación del estado mutacional del gen KRAS es una buena herramienta de pronóstico en aquellos pacientes con CCRm. Hoy en día se necesitan cada vez más estudios moleculares que permitan caracterizar mejor la heterogeneidad de la enfermedad con el fin de seleccionar un tratamiento óptimo ${ }^{[15]}$. La adición de anticuerpos monoclonales anti-EGFR a la quimioterapia mejora la supervivencia libre de progresión, la supervivencia global y la tasa de respuesta tumoral de aquellos pacientes con CCRm que presenten el exón
2 no mutado en el gen KRAS ${ }^{[16]}$; no obstante, dicha investigación no comparó los distintos tratamientos en función a la mutación o no del exón 2 del gen KRAS. Con la misma base de datos usada en el presente estudio, ya he publicado la evolución del CCRm con distintos tratamientos, independientemente del gen KRAS, las respuestas y las curvas de supervivencia, las mismas que coinciden notablemente con la data internacional ${ }^{[17]}$.

En el presente estudio se analiza el comportamiento de la expresión del exón 2 del gen KRAS en pacientes con CCRm, independientemente del tratamiento que tuvieron. El objetivo primario fue medir la supervivencia global en función de la mutación o no del gen KRAS en pacientes con CCRm; los objetivos secundarios fueron medir la supervivencia global según las características clínicas y estado mutacional del exón 2 del gen KRAS y la supervivencia global con KRAS mutado frente al no mutado con las diferentes alternativas terapéuticas.

\section{MATERIALES Y MÉTODOS}

\section{Diseño del estudio}

El presente es un estudio un estudio observacional, retrospectivo, longitudinal.

\section{Población y muestra}

Durante el periodo de enero de 2010 a diciembre del 2013 se obtuvieron las historias clínicas de pacientes con CCRm que tuvieron evaluación tumoral del gen KRAS en los principales hospitales de cáncer de Lima Metropolitana: EsSalud (hospitales Edgardo Rebagliati Martins y Guillermo Almenara Irigoyen), hospitales del Ministerio de Salud (Instituto de Enfermedades Neoplásicas), hospitales Militar Central del Perú y Central de la Fuerza Aérea del Perú y clínicas privadas de Lima Metropolitana.

\section{Criterios de selección}

Se revisaron las historias clínicas de los pacientes con diagnóstico de CCRm, cuyos tumores hubieran sido estudiados para la mutación del gen KRAS y que tuvieran un tiempo de seguimiento de al menos tres meses o, en su defecto, hasta la fecha en la que hubieran fallecido por causas relacionadas con el cáncer. Se excluyeron aquellas historias clínicas que no tuvieran los datos necesarios para realizar el estudio.

\section{Procedimientos y técnicas}

Se estudiaron las mutaciones del exón 2 (codones 12 y 13) del oncogén KRAS. Para la evaluación del estado mutacional se realizaron las siguientes técnicas moleculares: secuenciación directa (método de Sanger o pirosecuenciación), PCR (reacción en cadena de la polimerasa) cuantitativa en tiempo real, técnicas de enriquecimiento del alelo mutado, análisis de polimorfismos de fragmentos de restricción con PCR y HRM (high resolution melting).

\section{Plan de análisis}

Las evaluaciones estadísticas incluyeron tabulaciones descriptivas (medidas de resumen numérico y métodos gráficos) y se usó la 
Tabla 1. Características clínicas de pacientes con CCRm y KRAS mutados y no mutados.

\begin{tabular}{|c|c|c|c|c|}
\hline \multirow{3}{*}{ Variable } & \multirow{3}{*}{ Total } & \multicolumn{2}{|c|}{ Gen KRAS } & \multirow{3}{*}{ Valor de $p^{*}$} \\
\hline & & Mutado & No mutado & \\
\hline & & n (\%) & $n(\%)$ & \\
\hline Frecuencia & 320 & $93(29,1)$ & $227(70,93)$ & \\
\hline \multicolumn{5}{|l|}{ Edad (en años) } \\
\hline$<60$ & 134 & $33(35,5)$ & $101(44,5)$ & \multirow{2}{*}{0,174} \\
\hline$\geq 60$ & 186 & $60(64,5)$ & $126(55,5)$ & \\
\hline \multicolumn{5}{|l|}{ Sexo } \\
\hline Femenino & 146 & $38(40,9)$ & $108(47,6)$ & \multirow{2}{*}{0,331} \\
\hline Masculino & 174 & $55(59,1)$ & $119(52,4)$ & \\
\hline \multicolumn{5}{|l|}{ PS ECOG } \\
\hline $0-1$ & 284 & $84(90,3)$ & $200(88,1)$ & \multirow{2}{*}{0,708} \\
\hline 2 & 36 & $9(9,7)$ & $27(11,9)$ & \\
\hline \multicolumn{5}{|l|}{ Origen del CCRm } \\
\hline Colon & 200 & $58(62,4)$ & $142(62,6)$ & \multirow{2}{*}{0,482} \\
\hline Recto & 87 & $21(22,6)$ & $66(29,1)$ & \\
\hline \multicolumn{5}{|l|}{ Metástasis inicial } \\
\hline No & 128 & $37(39,8)$ & $91(40,1)$ & \multirow{2}{*}{1,000} \\
\hline Sí & 192 & $56(60,2)$ & $136(59,9)$ & \\
\hline \multicolumn{5}{|l|}{ Carcinomatosis } \\
\hline No & 265 & $73(78,5)$ & $192(84,6)$ & \multirow{2}{*}{0,335} \\
\hline Sí & 54 & $19(20,4)$ & $35(15,4)$ & \\
\hline \multicolumn{5}{|l|}{ Metástasis hígado } \\
\hline No & 131 & $44(47,3)$ & $87(38,3)$ & \multirow{2}{*}{0,151} \\
\hline Sí & 188 & $48(51,6)$ & $140(61,7)$ & \\
\hline \multicolumn{5}{|l|}{ Metástasis pulmón } \\
\hline No & 222 & $70(75,3)$ & $152(67)$ & \multirow{2}{*}{0,141} \\
\hline Sí & 97 & $22(23,7)$ & $75(33)$ & \\
\hline \multicolumn{5}{|c|}{ Grado de diferenciación } \\
\hline Bien & 35 & $12(19)$ & $23(13,7)$ & \multirow{4}{*}{0,277} \\
\hline Moderado & 168 & $41(65,1)$ & $127(75,6)$ & \\
\hline Pobre & 28 & $10(15,9)$ & $18(10,7)$ & \\
\hline Desconocido & 89 & $30(-)$ & $59(-)$ & \\
\hline \multicolumn{5}{|l|}{ Tratamiento previo } \\
\hline Ninguno & 172 & $56(61,5)$ & $116(52,5)$ & \multirow{4}{*}{0,342} \\
\hline Neoadyuvante & 33 & $8(8,8)$ & $25(11,3)$ & \\
\hline Adyuvante & 107 & $27(29,7)$ & $80(36,2)$ & \\
\hline No determinado & 8 & $2(-)$ & $6(-)$ & \\
\hline
\end{tabular}

PS ECOG (del inglés performance status ECOG): estado funcional del paciente de acuerdo con la escala de la European Clinical Oncology Group; CCRm: carcinoma colorrectal metastásico.

* obtenido mediante la prueba Chi cuadrado 


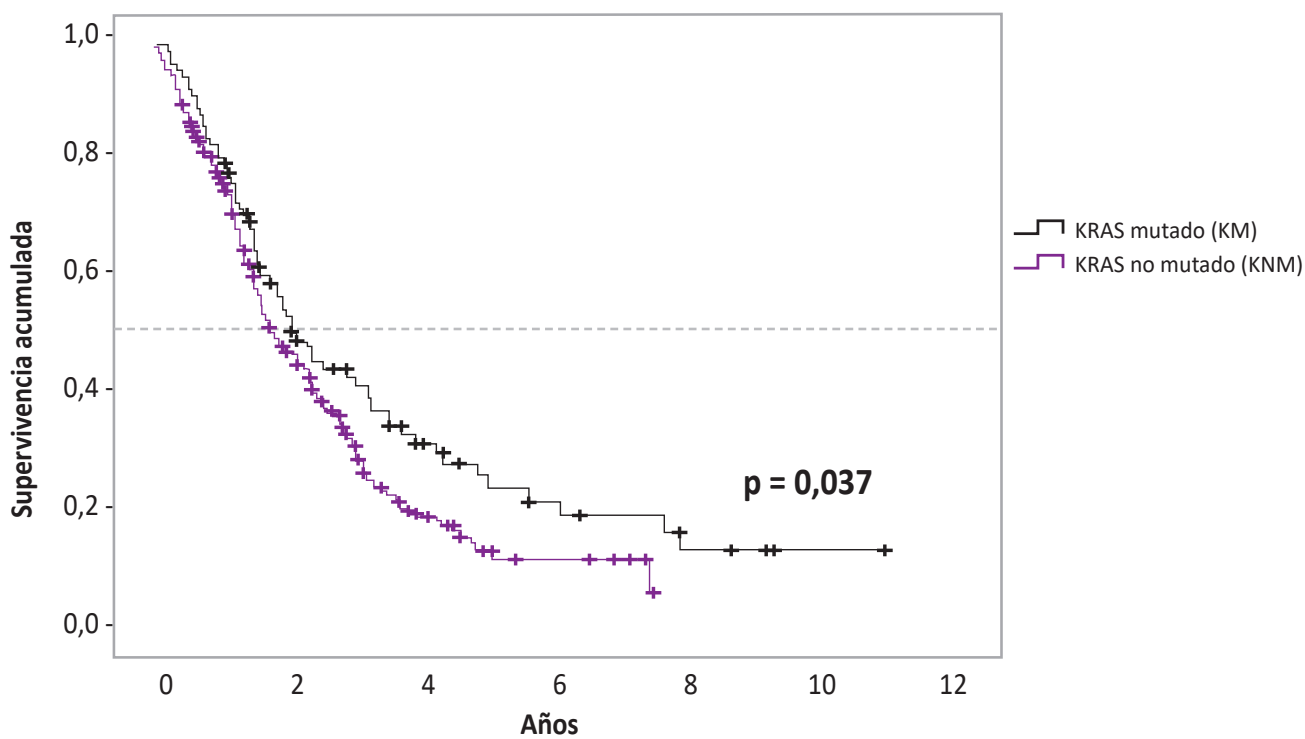

Figura 1. Supervivencia global de pacientes con CCRm con el exón 2 del gen KRAS mutado versus no mutado.

prueba chi cuadrado en la comparación. La supervivencia global se determinó desde la fecha de diagnóstico de la metástasis hasta la fecha de fallecimiento o fecha de último control.

Las curvas de supervivencia fueron estimadas mediante el método de Kaplan-Meier; las comparaciones de las curvas de sobrevivencia se realizaron mediante la prueba de long rank o Breslow. Para fines de interpretación se estimó la mediana de supervivencia, así como el hazard ratio (HR) y sus intervalos de confianza al 95 \% (IC 95\%). Toda la evaluación estadística se realizó con un nivel de significancia de $5 \%(p<0,05)$. Las evaluaciones estadísticas se realizaron mediante el programa SPSS (Versión 23).

\section{Consideraciones éticas}

El proyecto de investigación fue presentado y aprobado por el Comité Institucional de Ética de la Universidad Cayetano Heredia (inscripción Comité de Ética Nº6629).

\section{RESULTADOS}

Se revisaron 540 historias clínicas de pacientes con evaluación del estado mutacional del exón 2 del gen KRAS. Del total de historias clínicas revisadas se excluyeron 121 pacientes que tuvieron cáncer colorrectal no metastásico en su mayor parte, otras neoplasias malignas como cáncer de pulmón u otros cánceres gastrointestinales. De los 419 pacientes restantes con CCRm, hubo 99 casos que no cumplieron los criterios de inclusión (la mayor parte por información limitada de la historia clínica, por ejemplo, pasaron a otra institución para continuar el tratamiento o viajaron a su sitio de origen en provincias y no se pudo obtener la información requerida para el estudio). Por tanto, la población de evaluación final en el presente estudio fue de 320 pacientes con CCRm y estudio mutacional del gen KRAS en el exón 2.
La mediana de la edad del total de participantes fue de 63 años (rango: 15 a 90 años), la mediana de edad fue de 64 y 63 años en los pacientes con KRAS mutado y no mutado, respectivamente. Un total de 251 pacientes provinieron de Lima $(78,4 \%)$ y 68 de provincias (21,3\%). Respecto al sitio del origen del CCR, no se pudo determinar en 34 casos $(10,6 \%)$ y se catalogó como carcinomatosis peritoneal, en $65(20,3 \%)$ casos correspondió a colon ascendente, en $23(10,0 \%)$ a colon transverso, en 111 $(34,7 \%)$ a colon descendente y sigmoides, y en $87(27,2 \%)$ a recto. De total, 227 (70,93\%) pacientes tuvieron el gen KRAS no mutado o nativo y $93(29,07 \%)$ lo tuvieron. La diferencia de las características clínicas de los pacientes, en ambos grupos, no fue estadísticamente significativa (Tabla 1).

\section{Supervivencia global de pacientes con CCRm con KRAS} mutado frente a no mutado

Con un seguimiento promedio de 82,8 meses se evaluó la supervivencia global de los pacientes con base al estado mutacional del exón 2 del gen $K R A S$, al margen del tratamiento utilizado. Los pacientes con CCRm y con mutación del exón 2 del gen KRAS tuvieron una mediana de supervivencia global de 25,2 meses (IC 95\%: 18,3 -32, 0 meses) frente a aquellos con KRAS no mutado (mediana: 21,3 meses; IC 95\%: 16,6 - 25,9 meses). La tasa de supervivencia a los 2 y 5 años también fue mayor en el grupo con KRAS mutado (53,0\% y $23,6 \%$, respectivamente) frente a aquellos con KRAS no mutados (47,3\% y $12,9 \%$, respectivamente), siendo esta diferencia estadísticamente significativa (HR: 0,73; IC 95\%: 0,55-0,98; $p=0,037$ ) (Figura 1).

\section{Supervivencia global del CCRm según las características} clínicas y estado mutacional del exón 2 del gen KRAS

La mediana de supervivencia y la tasa de supervivencia a los cinco años de cada característica clínica en casi todos los casos favoreció al grupo con mutación del exón 2 del gen KRAS; 
Tabla 2. Supervivencia global del CCRm según las características clínicas y estado mutacional del exón 2 del gen KRAS

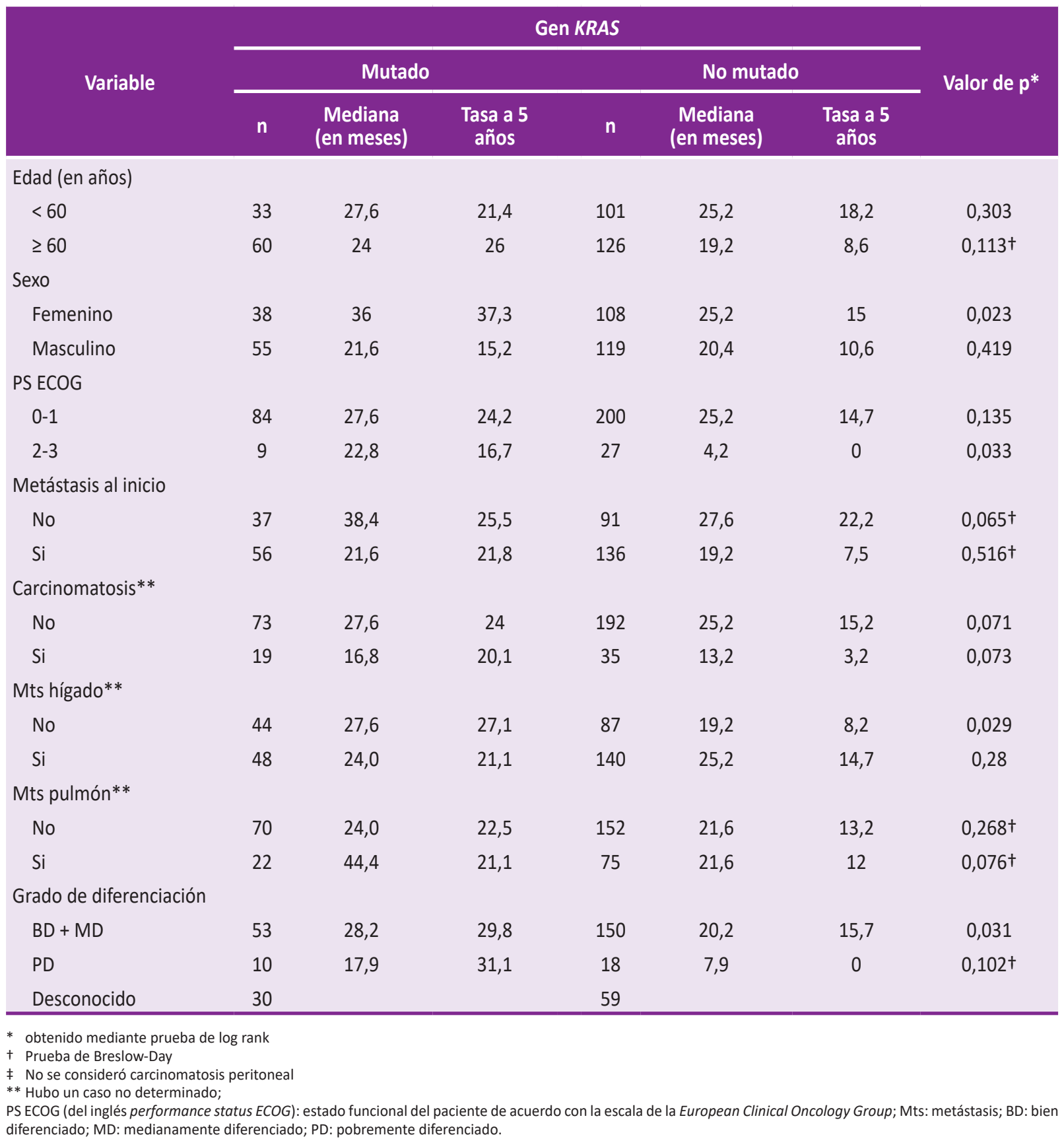

incluso en algunos con significancia estadística, lo cual explica el comportamiento de la curva de supervivencia global a favor de los pacientes con KRAS mutado (Tabla 2).

Supervivencia global de pacientes con CCRm con KRAS mutado versus no mutado con las diferentes alternativas terapéuticas

Se evaluó los pacientes con CCRm que tuvieron tratamiento con quimioterapia (con o sin tratamiento biológico [uso de anticuerpos monoclonales]) y a aquellos sometidos a metastasectomía, con relación al estado mutacional del exón 2 del gen KRAS. Dentro del primer grupo hubo una mejor mediana de supervivencia en los pacientes con KRAS mutado (23,4 meses; IC 95\%: 17,2 - 29,7 meses) frente a aquellos con KRAS no mutado (18,1 meses; IC 95\%: 15,9 - 20,3 meses), aunque sin significancia estadística (Figura $2 \mathrm{~A}$ y Tabla 3).

Los pacientes con metastasectomía y KRAS mutado tuvieron mejor mediana de supervivencia (94,2 meses; IC 95\%: 13,6 - 174,7 meses) que el grupo con KRAS no mutado (44,4 meses; IC 95\%: 34,5-54,2 meses); sin embargo, la diferencia en la mediana, así como las 
A Quimioterapia con y sin anticuerpos monoclonales

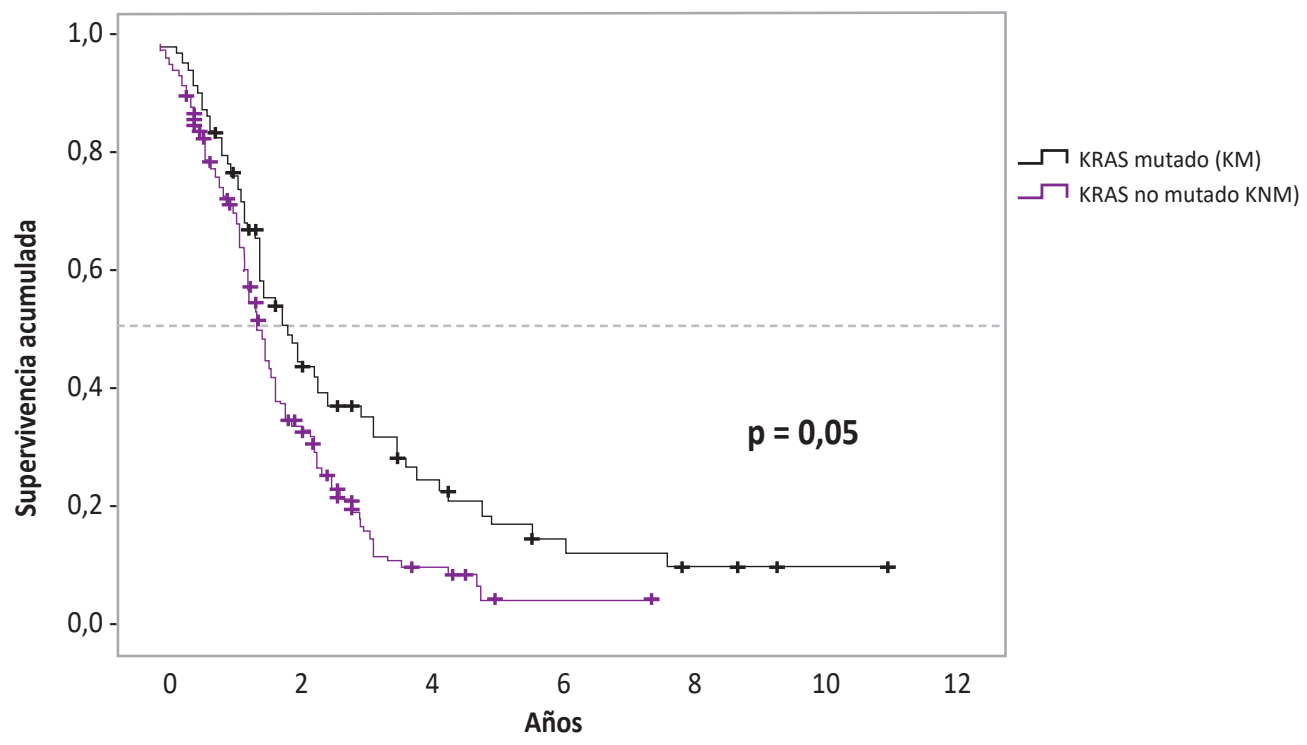

B. Metastasectomía

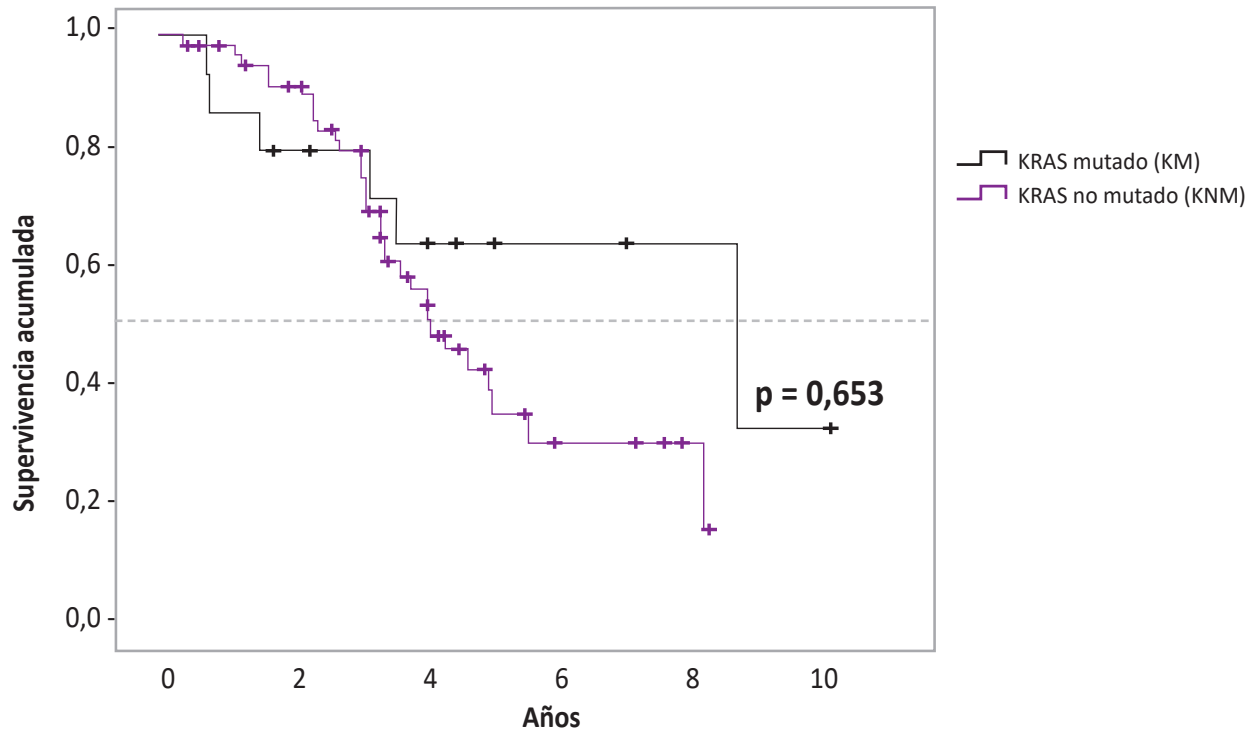

Figura 2. Supervivencia global de pacientes con CCRm con KRAS mutado versus no mutado con las diferentes alternativas terapéuticas.

diferencias en la supervivencia a dos y cinco años de supervivencia no fueron significativos $(0,653)$. En relación a los que recibieron quimioterapia o quimioterapia más anticuerpos monoclonales la mediana de supervivencia fue mayor para el grupo con K-ras mutado que para el no mutado $(23,4$ versus 18,1$)$ con un nivel de significancia de 0,005 (Figura 2B y Tabla 3).

\section{DISCUSIÓN}

La mutación del exón 2 del gen KRAS en CCRm se encontró entre el $35 \%$ y el $45 \%$ de los pacientes que ingresaron a diferentes estudios, sobre todo en Europa y EE. UU. ${ }^{[8-11]}$. En Asia, se identificó una tasa más baja de esta mutación; así en Taiwán se encontró el 26,5\% de KRAS mutado ${ }^{[18]}$. En este estudio el porcentaje de pacientes con CCRm con el exón 2 del gen KRAS mutado fue de 29,07\%; sin embargo, un reporte del año 2011 en el Instituto de Enfermedades Neoplásicas ${ }^{[19]}$ mostró para el mismo grupo una frecuencia del $16,7 \%$. Un estudio similar realizado en Venezuela encontró una frecuencia de mutación del KRAS de $23,3 \%{ }^{[20]}$, en Argentina del $28 \%{ }^{[21]}$ y en Brasil, con la serie más grande de toda Latinoamérica (8 234 pacientes con CCRm) de 31,9\%, con pocas variaciones por áreas geográficas ${ }^{[22]}$. Las diversas variaciones pueden ser debido a diferentes factores como el tipo racial, cultural, genético, etc.; sin embargo, la diferencia en la tasa de mutación del gen KRAS podría deberse a otros aspectos como el tejido de la biopsia, el porcentaje de células tumorales en la muestra, la calidad del ADN extraído y, sobre todo, a los métodos de ensayo empleados para evaluar la mutación genética ${ }^{[2,24]}$. 
Tabla 3. Supervivencia global de pacientes con CCRm con KRAS mutado versus no mutado con las diferentes alternativas terapéuticas.

\begin{tabular}{|c|c|c|c|c|}
\hline \multirow[t]{2}{*}{ Variable } & \multicolumn{2}{|c|}{$\begin{array}{c}\% \text { de } \\
\text { supervivencia }\end{array}$} & \multirow{2}{*}{$\begin{array}{l}\text { Mediana } \\
\text { (IC 95\%) }\end{array}$} & \multirow{2}{*}{$\begin{array}{l}\text { Valor } \\
\text { de } p\end{array}$} \\
\hline & 2 años & 5 años & & \\
\hline \multicolumn{5}{|l|}{ Metastectomía } \\
\hline Mutado ( $n=15$ ) & 80,0 & 64,0 & $94,2(13,6-174,7)$ & 0,653 \\
\hline $\begin{array}{l}\text { No mutado } \\
(n=61)\end{array}$ & 89,3 & 34,7 & $44,4(34,5-54,2)$ & \\
\hline \multicolumn{5}{|l|}{$\mathrm{Qt} / \mathrm{Qt}+\mathrm{AM}$} \\
\hline Mutado $(n=73)$ & 48,7 & 16,6 & $23,4(17,2-29,7)$ & 0,005 \\
\hline $\begin{array}{l}\text { No mutado } \\
(\mathrm{n}=150)\end{array}$ & 35,0 & 4,2 & $18,1(15,9-20,3)$ & \\
\hline
\end{tabular}

CCRm: carcinoma colorrectal con metástasis; Qt: quimioterapia; AM: anticuerpos monoclonales; IC 95\%: intervalo de confianza al 95\%.

Llama la atención una mayor tasa de supervivencia en las mujeres; sin embargo, la diferencia en otros parámetros como el estado funcional del paciente, cáncer en lado derecho y transverso versus el lado izquierdo, ausencia de metástasis inicial versus metástasis al diagnóstico, presencia de carcinomatosis y el grado de diferenciación, son congruentes con lo reportado en la literatura mundial. En pacientes jóvenes, se reporta que las mujeres con CCRm sobreviven más que los hombres y se consideran que el estado hormonal desempeñaría un papel importante no solo en el desarrollo y la patogénesis del CCR, sino que puede tener una importancia pronóstica ${ }^{[25]}$.

Con respecto a la supervivencia global de pacientes con CCRm con KRAS mutado versus no mutado, contrariamente a lo reportado en la literatura médica, el grupo con mutación tuvo una mediana de supervivencia global superior al sin mutación. Respecto a la consideración del KRAS mutado como factor pronóstico negativo en CCR, ha habido muchas discrepancias con algunos investigadores que hallaron que la mutación KRAS era un factor pronóstico independiente ${ }^{[26]}$, mientras otros mostraban lo contrario [27]; empero, el estudio RASCAL, que incluyó datos primarios de 2721 pacientes con CCR de 22 grupos de investigación en 13 países diferentes, encontró que la presencia de la mutación KRAS se asoció significativamente con un peor pronóstico ${ }^{[28]}$. En nuestro estudio, los resultados fueron contradictorios con este último, probablemente debido factores étnicos, laboratoriales, respuesta a tratamiento o por el diseño de estudio (estudio no prospectivo ni controlado).

Finalmente, la frecuencia de realización de pruebas de KRAS ha estado aumentando en todo el mundo; sin embargo, en países desarrollados, aproximadamente el $50 \%$ de los pacientes que serían elegibles para dicho estudio no se lo realizaron por diferentes motivos ${ }^{[29]}$. Probablemente en el futuro, las biopsias líquidas buscando células tumorales circulantes nos entreguen más alternativas para tener biomarcadores predictivos para decidir el tratamiento más adecuado ${ }^{[30]}$.
Este estudio presenta limitaciones. Al tener un carácter retrospectivo, no se pudo establecer una adecuada comparación de grupos por los diferentes sesgos asociados a este diseño. Además, las pruebas de laboratorio usadas no fueron certificadas por algún organismo nacional que garantice que los resultados fueran fiables.

Se recomienda que los estudios para la evaluación y tratamiento, sobre todo aquellos moleculares usados para estudiar cáncer, estén estandarizados, así como los laboratorios que los procesan deberían tener certificados con referentes mundiales ${ }^{[31]}$. Debiera establecerse una vigilancia activa a nivel de cada hospital especializado y bajo la responsabilidad de las autoridades regulatorias de salud, para que los exámenes de este tipo sean confiables. El manejo del tratamiento del cáncer debería monitorearse continuamente basarse en guías de tratamiento con niveles de evidencia suficientes que apoyen las medidas terapéuticas que se ofrezcan.

Se concluye que la supervivencia global de pacientes con CCRm con KRAS mutado en Lima Metropolitana fue significativamente mejor que aquellos sin mutación; la revisión de la supervivencia por subgrupos basados en las características clínicas y estado mutacional del exón 2 del gen KRAS confirmaron estos hallazgos. La supervivencia global por modalidad terapéutica asociada al estado mutacional del exón 2 del gen KRAS también resultó en una mejor supervivencia del grupo mutado versus el no mutado. Son hallazgos diferentes a lo reportado en la literatura mundial (grupo KRAS no mutado presenta mejores pronóstico y respuesta al tratamiento).

Contribuciones de autoría. FA realizó la concepción y diseño del trabajo, recolección y/o obtención de los datos, análisis e interpretación de los resultados, redacción del manuscrito y aprobación de la versión final del artículo.

Potenciales conflictos de interés: ninguno.

Fuente de financiamiento. autofinanciado.

\section{ORCID:}

Franklin Aldecoa, https://orcid.org/0000-0003-3401-3689

\section{REFERENCIAS BIBLIOGRÁFICAS}

1. Payet E, Pérez P, Poquioma E, Díaz E, editores. Registro de cáncer de Lima Metropolitana. Incidencia y Mortalidad 2010 - 2012[Internet]. Volumen V. Lima: INEN; 2016 [citado 20 de enero 2020]. Disponible en: https://www.inen.sld.pe/portal/documentos/ pdf/banners_2014/2016/Registro\%20de\%20C\%C3\%A1ncer\%20 Lima\%20Metropolitana\%202010\%20-\%202012_02092016.pdf.

2. Van der Geest LGM, Lam-Boer J, Koopman M, Verhoef C, Elferink MAG, De Wilt JHW. Nationwide trends in incidence, treatment and survival of colorectal cancer patients with synchronous metastases. Clin Exp Metastasis. 2015;32(5):457-65. doi: 10.1007/s10585-0159719-0. 
3. Andres A, Mentha G, Adam R, Gerstel E, Skipenko OG, Barroso E, et al. Surgical management of patients with colorectal cancer and simultaneous liver and lung metastases. Br J Surg. 2015;102(6):6919. doi: 10.1002/bjs.9783.

4. Krasinskas AM. EGFR signaling in colorectal carcinoma. Patholog Res Int. 2011:932932. doi: 10.4061/2011/932932.

5. Karapetis CS, Khambata-Ford S, Jonker DJ, O'Callaghan CJ, Tu D, Tebbutt NC, et al. K-ras mutations and benefit from cetuximab in advanced colorectal cancer. N Engl J Med. 2008;359(17):1757-65.

6. Martins M, Mansinho A, Cruz-Duarte R, Martins SL, Costa L. AntiEGFR therapy to treat metastatic colorectal cancer: not for all. Adv Exp Med Biol. 2018;1110:113-31. doi: 10.1007/978-3-030-02771$1 \_8$.

7. Steele CW, Whittle T, Smith JJ. Review: KRAS mutations are influential in driving hepatic metastases and predicting outcome in colorectal cancer. Chin Clin Oncol. 2019;8(5):53. doi: 10.21037/ cco.2019.08.16

8. Amado RG, Wolf M, Peeters M, Van Cutsem E, Siena S, Freeman DJ, et al. Wild-type K-RAS is required for panitumumab efficacy in patients with metastatic colorectal cancer. J Clin Oncol. 2008;26(10):1626-34. doi: 10.1200/JCO.2007.14.7116.

9. Bokemeyer C, Bondarenko I, Makhson A, Hartmann JT, Aparicio $\mathrm{J}$, de Braud $\mathrm{F}$ et al. Fluorouracil, leucovorin, and oxaliplatin with and without cetuximab in the first line treatment of metastatic colorectal cancer. J Clin Oncol. 2009;27(5):663-71. doi: 10.1200/ JCO.2008.20.8397.

10. Peeters M, Price TJ, Cervantes A, Sobrero AF, Ducreux M, Hotko $Y$, et al. Randomized phase III study of panitumumab with fluorouracil, leucovorin, and irinotecan (FOLFIRI) compared with FOLFIRI alone as second-line treatment in patients with metastatic colorectal cancer. J Clin Oncol. 2010;28(31):4706-13. doi: 10.1200/ JCO.2009.27.6055.

11. Douillard JY, Siena S, Cassidy J, Tabernero J, Burkes R, Barugel M, et al. Randomized, phase III trial of panitumumab with infusional fluorouracil, leucovorin, and oxaliplatin (FOLFOX4) versus FOLFOX4 alone as first-line treatment in patients with previously untreated metastatic colorectal cancer: the PRIME study. J Clin Oncol. 2010;28(31):4697-705. doi: 10.1200/JCO.2009.27.4860.

12. Stintzing $S$, Modest DP, Rossius L, Lerch MM, von Weikersthal LF, Decker T, et al. FOLFIRI plus cetuximab versus FOLFIRI plus bevacizumab for metastatic colorectal cancer (FIRE-3): a post-hoc analysis of tumour dynamics in the final RAS wild-type subgroup of this randomised open-label phase 3 trial. Lancet Oncol. 2016;17(10):1426-34. doi: 10.1016/\$1470-2045(16)30269-8.

13. Maffeis V, Nicolè L, Cappellesso R. RAS, cellular plasticity, and tumor budding in colorectal cancer. Front Oncol. 2019;9:1255. doi: 10.3389/fonc.2019.01255

14. Porru M, Pompili L, Caruso C, Biroccio A, Leonetti C. Targeting KRAS in metastatic colorectal cancer: current strategies and emerging opportunities. J Exp Clin Cancer Res. 2018;37(1):57. doi: 10.1186/ s13046-018-0719-1.

15. Rasmy A, Fayed A, Omar A, Fahmy N. Effect of KRAS mutational status on disease behavior and treatment outcome in patients with metastatic colorectal cancer: intratumor heterogeneity and mutational status. J Gastrointest Oncol. 2019;10(5):886-95. doi: 10.21037/jgo.2019.05.04

16. Chan DLH, Segelov E, Wong RS, Smith A, Herbertson RA, Li $\mathrm{BT}$, et al. Epidermal growth factor receptor (EGFR) inhibitors for metastatic colorectal cancer. Cochrane Database Syst Rev. 2017;6(6):CD007047. doi: 10.1002/14651858.CD007047.pub2.
17. Aldecoa, F. Cáncer colorrectal metastásico: supervivencia global con diferentes alternativas de tratamiento en Lima Metropolitana. Acta Med Peru. 2019;36(3):195-201. doi: 10.35663/amp.2019.363.828

18. Wu CM, Tang R, Wang JY, Changchien CR, Hsieh LL. Frequency and spectrum of K-RAS codons 12 and 13 mutations in colorectal adenocarcinomas from Taiwan. Cancer Genet Cytogenet. 2005;158(1):55-60. doi: 10.1016/j.cancergencyto.2004.08.030

19. Egoavil CM, Montenegro P, Soto JL, Casanova L, Sanchez-Lihon J, Castillejo Ml, et al. Clinically important molecular features of Peruvian colorectal tumours: high prevalence of DNA mismatch repair deficiency and low incidence of K-RAS mutations. Pathology. 2011;43(3):228-33. doi: 10.1097/PAT.0b013e3283437613.

20. Estrada P, Rojas-Atencio A, Zabala W, Borjas L, Soca L, Urdaneta K, et al. Frecuencia y asociación clínico patológico de las mutaciones del oncogén K-ras en pacientes venezolanos con cáncer colorectal. Invest Clin. 2009;50(1):55-63.

21. Redal MA, Perez MPI. Tipificación molecular de K-ras en cáncer colorrectal metastásico. Poster presentado en: IV Congreso de médicos en formación en oncología. 2010 nov 19-20; Buenos Aires, Argentina.

22. Gil C, Aran V, Zalcberg-Renault I, Victorino AP, Salem JH, Bonamino $\mathrm{MH}$, et al. K-RAS mutations: variable incidences in a Brazilian cohort of 8,234 metastatic colorectal cancer patients. BMC Gastroenterology. 2014;14:73. doi: 10.1186/1471-230X-14-73.

23. Tan C, Du X. K-RAS mutation testing in metastatic colorectal cancer. World J Gastroenterol. 2012;18(37):5171-80. doi: 10.3748/wjg.v18. i37.5171.

24. Matsunaga M, Kaneta T, Miwa K, Ichikawa W, Fujita KI, Nagashima F, et al. A comparison of four methods for detecting KRAS mutations in formalin-fixed specimens from metastatic colorectal cancer patients. Oncol Lett. 2016;12(1):150-6. doi: 10.3892/ol.2016.4576.

25. Hendifar A, Yang D, Lenz F, Lurje G, Pohl A, Lenz C, et al. Gender disparities in metastatic colorectal cancer survival. Clin Cancer Res.2009; Oct 15;15(20):6391-7. doi: 10.1158/1078-0432.CCR-090877.

26. Tanaka M, Omura K, Watanabe Y, Oda Y, Nakanishi I. Prognostic factors of colorectal-cancer: K-ras mutation, overexpression of the p53-protein, and cell proliferative activity. J Surg Oncol. 1994;57(1):57-64. doi: 10.1002/jso.2930570115.

27. Dix BR, Robbins $P$, Soong R, Jenner D, House AK, lacopetta BJ. The common molecular genetic alterations in Dukes' $B$ and $C$ colorectal carcinomas are not short-term prognostic indicators of survival. Int J Cancer. 1994;59(6):747-51. doi: 10.1002/ijc.2910590606.

28. Andreyev HJ, Norman AR, Cunningham D, Oates JR, Clarke PA. Kirsten Ras mutations in patients with colorectal cancer: the multicenter "RASCAL" study. J Natl Cancer Inst. 1998;90(9):675-84 doi: 10.1093/jnci/90.9.675.

29. Carter GC, Landsman-Blumberg PB, Johnson BH, Juneau P, Nicol SJ, $L i$, et al. KRAS testing of patients with metastatic colorectal cancer in a community-based oncology setting: a retrospective database analysis. J Exp Clin Cancer Res. 2015;34(1):29. doi: 10.1186/s13046015-0146-5.

30. Kondo $\mathrm{Y}$, Hayashi K, Kawakami K, Miwa Y, Hayashi H, Yamamoto M. KRAS mutation analysis of single circulating tumor cells from patients with metastatic colorectal cancer. BMC Cancer. 2017;17(1):311. doi: 10.1186/s12885-017-3305-6.

31. Hernández-Losa J, Sanz J, Landolfi S, López-Ríos F, Palacios J, Bautista $\mathrm{MD}$, et al. Recomendaciones para la determinación de mutaciones de K-RAS en cáncer de colon. Rev Esp Patol. 2012;45(2):76-85. doi: 10.1016/j.patol.2011.11.005. 